\title{
Effects of observer variation in population screening for cervical carcinoma
}

\author{
A. LAMBOURNE AND H. LEDERER \\ From the Department of Community Medicine, University of Sheffield, and the Department of Pathology, \\ Doncaster Royal Infirmary, Doncaster, Yorkshire
}

SYNOPSIS A stratified random sample of 100 cervical smears was taken from routine population screening material and graded by three cytological laboratories. The sensitivity of the smear test for detecting cervical malignancies varied from 72 to $88 \%$-a difference of $16 \%$; for all severe epithelial abnormalities the sensitivity varied from 52 to $71 \%$ (a difference of $19 \%$ ). The histological material pertaining to these smears was re-examined 'blindly' and then sent for diagnosis to a second pathologist. Using each set of histodiagnoses as reference, the sensitivity of the test for detecting cervical malignancies varied from 77 to $85 \%$-a difference of $8 \%$; the sensitivity for all epithelial abnormalities varied from 62 to $76 \%$ (a difference of $14 \%$ ). The significance of these observer variations on the efficacy of screening for cervical carcinoma is discussed.

Results of screening by cervical cytology recall strategy, and the histopathology of gynaecological operation specimens in a district hospital serving a population of 250000 over a period of five years have been analysed by Lederer and Lambourne (1973). In this communication variations by different observers in the grading of cervical smears and of histopathological findings in operation specimens of the same series are analysed in an attempt to determine how observer variation may affect estimates of the efficiency of cervical mass screening. The methods of investigation have also been detailed.

In order to assess observer variation, Evans (1970) sent the same batch of 100 smears to five cytology centres in Britain and compared their findings with his own. Having graded the smears into four classes, he found total disagreement with his diagnosis in $2 \%$, grading differences of two classes in $6 \%$, and of one class in $20 \%$ of the smears. Seybolt and Johnson (1971) submitted smears of 25 cases with doubtful cytological findings to eight 'leaders in the field of diagnostic cytology', and found marked diversity of interpretation, although, in the authors' opinion, only 18 of the 200 reports would have led to less than optimal management of the cases. Cocker, Fox, and Langley (1968) reported serious inconsistencies in histodiagnosis, not only between different pathologists, but also by the same pathologist after an interval of time.

Received for publication 12 June 1973.
The efficiency of a test in detecting epithelial abnormalities may be measured by means of its sensitivity, ie, the proportion of a particular abnormality discovered by the test. (Another measure commonly used is the 'false negative error rate', which is the complement of the sensitivity, ie, the proportion of conditions not discovered by the test.) The sensitivity of the cervical smear test is dependent on two separate factors: (1) the accuracy of the information recorded on the smear, and (2) the interpretation of both the smear and the confirmatory histological specimen.

Errors inherent in both of these factors were observed by Martin (1972) on examining the records of the 76 cases of invasive cervical carcinoma identified in San Diego, California, in 1967. In 33 of the 76 cases $(43 \%)$ a cytological smear obtained within a year had been reported as negative. Review of the slides was not possible for 20 of the 33 cases, but of the 13 cases whose slides had not been discarded, a review sustained the original negative report in three cases (finding inadequate cellular material) but changed the cytological diagnosis from negative to positive or suspicious in the remaining 10. In one invasive carcinoma, the histological specimen had been incorrectly read.

The authors felt that differences in criteria for interpreting both cytological and histological specimens may be partly responsible for variations in estimates of the sensitivity of the smear test. Thus the following trial was carried out in order to assess 
differences in grading of both cytological and histological specimens.

\section{Divergences in Cytological Grading}

\section{METHOD}

A stratified random sample of 100 smears was selected so that normal, atypical, and malignant smears were approximately equally represented. The batch thus included a grossly higher proportion of abnormal smears than would be obtained from a mass screening programme.

The smears were re-examined 'blindly' at the district hospital (referred to as D in the tables) and then sent to two well known cytology laboratories (referred to as A and B). All three centres used the same Papanicolau grading, classing the smears as I, II, III, or IV.

\section{RESULTS}

The findings of the three centres are summarized in table I which shows that $\mathbf{A}$ was more severe in

\begin{tabular}{lccc}
\hline Grade & \multicolumn{2}{l}{ Cytologist } & \\
\cline { 2 - 4 } & $D$ & $A$ & \multicolumn{1}{l}{$B$} \\
\hline IV & 23 & 37 & 23 \\
III & 18 & 16 & 17 \\
II & 28 & 39 & 29 \\
I & 30 & 7 & 25 \\
Unsatisfactory & 1 & 1 & 6 \\
Total & 100 & 100 & 100 \\
\hline
\end{tabular}

Table I Grading of smears

grading than either $\mathbf{B}$ or $\mathbf{D}$, classing more smears as grade IV and fewer as grade I, and apparent close agreement in grading between $\mathbf{D}$ and $\mathbf{B}$. The gradings for each pair of observers are analysed in table II and their differences shown in figure 1 . These show that $\mathbf{A}$ was more severe than $\mathbf{B}$ or $\mathbf{D}$ and that $\mathbf{B}$ and $D$ were not as consistent as might be assumed from table I, their gradings differing by one class or more in $37 \%$ of cases.

A statistic which summarizes the differences

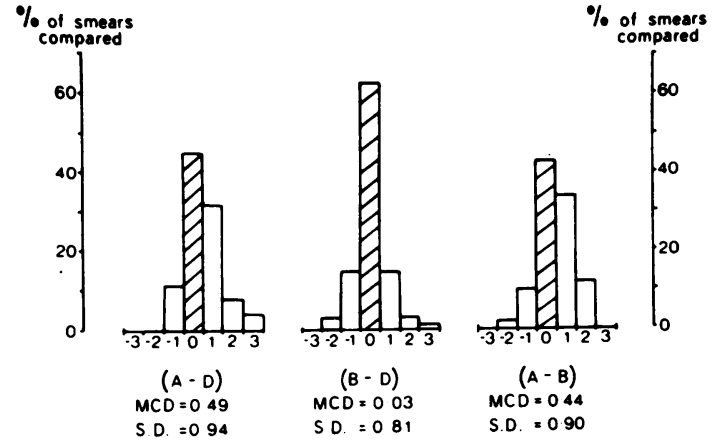

Fig 1 Pair-wise comparisons of cytological diagnoses.

between a pair of observers is the mean class difference (MCD)-the mean number of classes by which the grading of one observer differs from that of another; thus a MCD of +1 between $X$ and $Y$ indicates that on average a class graded as $N+1$ by $\mathrm{X}$ is graded as $\mathbf{N}$ by $\mathrm{Y}$. The MCD between $\mathrm{B}$ and $\mathrm{D}$ was low (0.03); those between both $A$ and $D$ and $A$ and $B$ were somewhat higher $(0.49$ and 0.44 respectively).

While the MCD measures the overall differences in grading between two observers, it gives no indication to their consistency; thus a MCD of +1 may be obtained from either a consistent set of differences $(1,1,1,1,1)$ or a random set $(0,3,-2,3,1)$. The standard deviation (SD) of the class difference variable is a measure of consistency, a low SD indicating good and a high SD poor consistency.

The consistency between each pair of cytologists was only moderate, the SD of the grading differences ranging from 0.81 to 0.94 . This is important for the differences between $B$ and $D$, since it shows that their low MCD was the result of balance between their differences rather than perfect agreement.

\section{Divergences in Histological Grading}

METHOD

In order to assess the effect of differences in grading

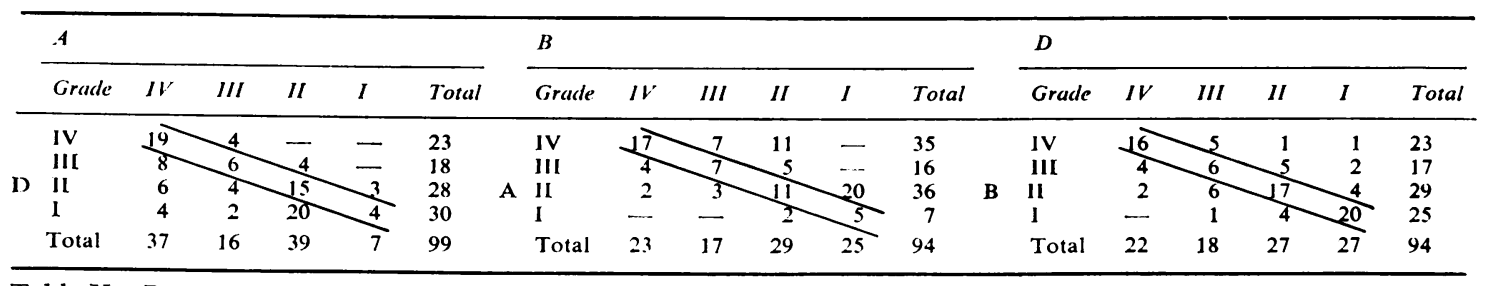

Table II Pair-u'ise comparison of cytological diagnoses ${ }^{1}$

${ }^{1}$ Numbers within the diagonal lines represent complete agreement between the two diagnoses 
of the confirmatory operation specimens, the histological material pertaining to the 100 cervical smears was examined. As some of these smears had been obtained from the same patient, biopsies of only 80 cases were available. These had been examined by one of the authors (H.L.) during 196670 using diagnostic criteria based on the work of Koss (1961) and at a later phase on the work of the panel of the Royal College of Obstetrics and Gynaecology (Govan, Haines, Langley, Taylor, and Woodcock, 1969). They were re-examined 'blindly' by him in 1971, using Govan's criteria, and 45 were sent to a second pathologist whose criteria were also based on Govan's article. Micro-invasive carcinoma was classed as 'invasive' in the analysis.

\section{RESULTS}

The original gradings of the author (O), his review gradings (D), and those of the second pathologist (A) are shown in table III. This suggests that the review gradings (D) were less severe than the original $(\mathrm{O})$ and that A's grading was less severe than both. The data were in a similar form to those of the cytological gradings, ie, four ranked classes, and so the same methods of statistical analysis were used. The results are given in table IV and figure 2. There was only a small difference in severity of grading between the original (O) and the review (D) gradings (MCD $=-0 \cdot 15$ ), while $A$ was less severe than both $O$ (by 0.60 classes) and $D$ (by 0.44 classes). The lower standard deviation of the D-O class

\begin{tabular}{llrrr}
\hline Grade & Description & \multicolumn{3}{c}{ Histologist } \\
\cline { 3 - 5 } & & $O$ & $D$ & $A$ \\
\hline III & Invasive servical carcinoma & 13 & 11 & 6 \\
II & In-situ carcinoma & 20 & 15 & 6 \\
I & Severe dysplasia & 25 & 29 & 16 \\
0 & Normal or mild dysplasia & 20 & 23 & 16 \\
- & Carcinoma of body of uterus & 2 & 2 & 1 \\
& Total & 80 & 80 & 45 \\
\hline
\end{tabular}

Table III Grading of histology specimens

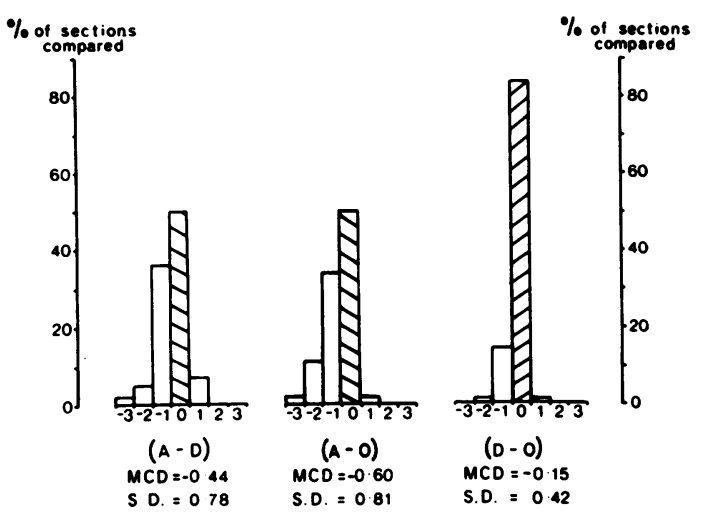

Fig 2 Pair-wise comparisons of histological diagnoses.

differences indicates a greater consistency in classification by the one pathologist after a lapse of time than between the two observers.

\section{Effects of Grading Differences on Estimates of the Sensitivity of the Test}

DIFFERENCES IN CYTOLOGICAL GRADING

In order to assess how these differences affect the estimates of sensitivity of the smear test, the cytodiagnoses of $\mathrm{A}, \mathrm{B}$, and $\mathrm{D}$ were compared with the histodiagnosis of $\mathbf{D}$ for the 72 samples for which all the required information was available. The cytodiagnoses were grouped as I or II and III or IV, since cases in the latter group would be submitted to follow up or operation, while no immediate action would be taken with those classed as I or II. The results are shown in tables $\mathrm{V}$ and VI.

In the 25 cases of in-situ or invasive carcinoma, action would have been taken in 18, 21 , or 22 cases on the basis of the cytodiagnoses of $\mathrm{D}, \mathrm{A}$, and $\mathrm{B}$ respectively. Thus the sensitivity of the test for all malignancies, namely, the proportion of invasive

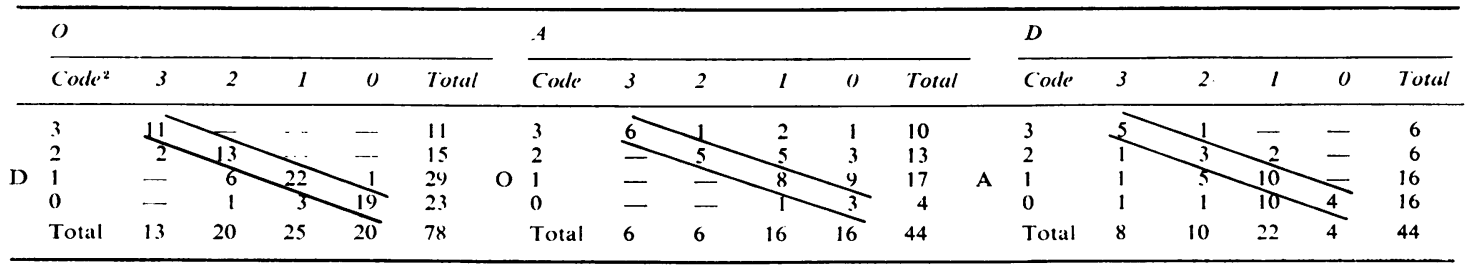

Table IV Pair-wise comparisons of histological diagnoses ${ }^{1}$

${ }^{1}$ Numbers within the diagonal lines represent complete agreement between the two diagnoses

3 = invasive cervical carcinoma, 2 = carcinoma in situ, $1=$ severe dysplasia $0=$ mild dysplasia or normal 


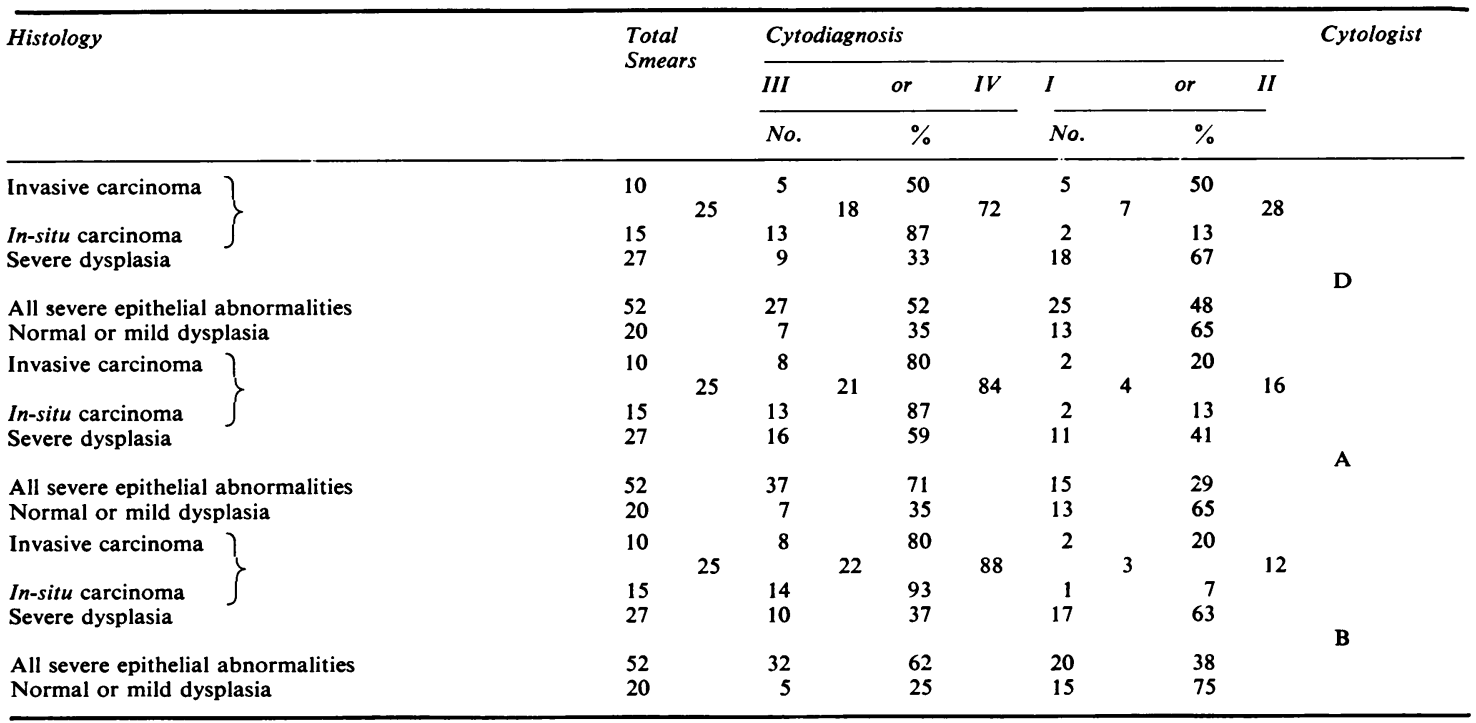

Table V Variations in cytological diagnoses

\begin{tabular}{lllllc}
\hline Conditions Detected & \multicolumn{2}{l}{ Cytologist } & & Mean Sensitivity (\%) & \\
\cline { 2 - 5 } & D & Maximum Variation \\
\hline Invasive and in-situ carcinoma & 72 & 84 & 88 & 81 & 16 \\
In-sttu carcinoma & 87 & 87 & 93 & 89 & 6 \\
All severe epithelial abnormalities & 52 & 71 & 62 & 62 & 19 \\
\hline
\end{tabular}

Table VI Variations in sensitivity caused by differences in cytological grading

and in-situ carcinomas detected by cytology varied from $18 / 25(72 \%)$ by D to $22 / 25(88 \%)$ by B, a difference of $16 \%$.

As the primary aim of the smear test is to discover precursors of invasive carcinoma, the sensitivity is often defined as the proportion of in-situ carcinomas detected (Spriggs, 1972). Of the 15 cases classified histologically as carcinoma in-situ, 13 or 14 were graded as III or IV by the three cytologists, giving sensitivities of 87 or $93 \%$ respectively-a variation of only $6 \%$.

On combining severe dysplasia, carcinoma in-situ, and invasive carcinoma, and defining sensitivity as the proportion of these conditions detected, the sensitivity fell quite substantially, as between 11 and 18 of the 27 cases of severe dysplasia were graded cytologically as I or II. The sensitivity of the test in detecting abnormalities more severe than mild dysplasia thus varied from 52 to $71 \%$-a variation of $19 \%$.

The analysis also shows that in 14 to $21 \%$ of the smears graded as III or IV, histological examination revealed no more significant lesion than mild dysplasia.
DIFFERENCES IN HISTOLOGICAL GRADING

To assess the variations in sensitivity caused by differences in histological grading, the cytodiagnoses were compared in turn with the original gradings $(O)$, the review gradings (D), and those of the second pathologist (A). In order to use the maximum information available, all three sets of cytodiagnoses were used, giving three cytological gradings for each of the 42 operation specimens examined. The results are shown in tables VII and VIII.

Of the 69 cytodiagnoses of the cases for which $O$ returned a histological report of a cervical malignancy, 53 had been graded as III or IV, giving a sensitivity of $53 / 69(77 \%)$. The sensitivity using D's histodiagnosis was $46 / 54(85 \%)$ and that of A was $28 / 36(78 \%)$. The proportion of cases warranting further treatment missed by the test thus varied from 15 to $23 \%$.

These figures are more consistent than those of the cytodiagnoses, but still produce a difference of $8 \%$ in the estimates of the test's sensitivity.

If, as before, we were to consider only the detection of in-situ carcinoma, the sensitivity varied by 18 from $72 \%$ by both $\mathrm{O}$ and $\mathrm{A}$ to $90 \%$ by $\mathrm{D}$. 


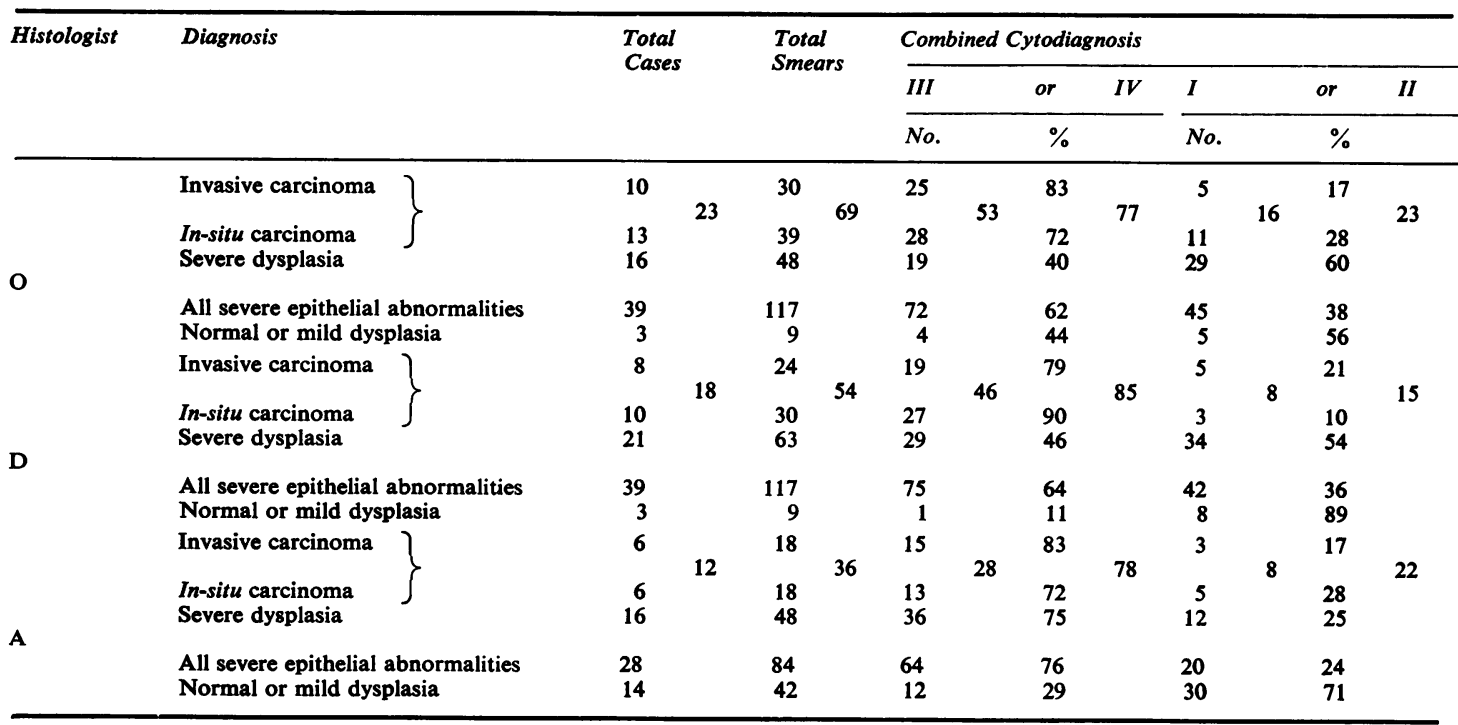

Table VII Variations in histological diagnoses

\begin{tabular}{|c|c|c|c|c|c|}
\hline \multirow[t]{2}{*}{ Conditions Detected } & \multicolumn{3}{|c|}{ Histologist } & \multirow[t]{2}{*}{ Mean Sensitivity (\%) } & \multirow[t]{2}{*}{ Maximum Variation } \\
\hline & $O$ & $\boldsymbol{D}$ & $A$ & & \\
\hline $\begin{array}{l}\text { Invasive and in-situ carcinoma } \\
\text { In-situ carcinoma } \\
\text { All severe epithelial abnormalities }\end{array}$ & $\begin{array}{l}77 \\
72 \\
62\end{array}$ & $\begin{array}{l}85 \\
90 \\
64\end{array}$ & $\begin{array}{l}78 \\
72 \\
76\end{array}$ & $\begin{array}{l}80 \\
78 \\
66\end{array}$ & $\begin{array}{r}8 \\
18 \\
14\end{array}$ \\
\hline
\end{tabular}

Table VIII Variations in sensitivity caused by differences in histological grading

Considering all severe epithelial abnormalities, the sensitivity fell to $62 \%(\mathrm{O}), 64 \%$ (D), or $76 \%$ (A)a variation of $14 \%$.

Thus, variations in histopathological grading both between different pathologists and the same pathologist after a lapse of time produced variations in the estimates of the test's sensitivity of $8 \%$ for detecting both invasive and in-situ carcinoma, $18 \%$ for in-situ carcinoma alone, and $14 \%$ for epithelial abnormalities more severe than mild dysplasia.

\section{Discussion}

Although population screening for cervical cancer has been common practice in Great Britain for over 10 years and for much longer in parts of North America, doubts on the value of screening programmes have been expressed mainly by epidemiologists on the basis of analysis of mortality and morbidity statistics.

Thus Ashluwalia and Doll (1968) pointed out that a comparable fall in incidence and mortality had occurred in parts of Canada not affected by the British Columbia screening programme (Boyes,
1969; Boyes, Worth, and Fidler, 1970). The conclusion of Mcgregor, Fraser, and Mann (1971) that a fall in incidence of invasive carcinoma in Aberdeen was attributable to the local screening programme has been criticized by Wilson, Chamberlain, and Cochrane (1971) and similar criticism seems valid for the report by Dickinson, Mussey, Soule, and Kurland (1972) from Rochester. Pedersen, Hoeg, and Kolstad (1971) in a detailed analysis of the experimental programme carried out by the Norwegian Cancer Society in the county of Østfold show that although the programme achieved a more favourable stage distribution of invasive carcinomas, there was no reduction in incidence of invasive carcinoma although hundreds of cases of dysplasia and carcinoma in-situ had been eliminated. A similar fall in stages II and III cases had occurred in the rest of Norway. They attribute the shortcomings of the programme to (a) failure to reach the high risk social class of the population; (b) the rapid development of some cervical cancers, which remain undetected unless the screening is carried out at impracticably short intervals; (c) observer and sampling errors inherent in the test which allow 
lesions to pass undetected. Koss (1972) refers to the problem of differences in terminology and observer variation in the detection of cervical carcinoma by cytology.

We have in this analysis substituted the vague term 'false error rate' by the concept of sensitivity of the test, which allows analysis of diagnoses of various degrees of epithelial abnormality. This seemed important as different preventative and therapeutic procedures have been based on frequently loosely defined morphological criteria. Although in their investigations both cytologists and histologists used the same clearly defined criteria and although the senior technicians in Laboratory D responsible for screening had originally been trained in laboratory A, significant observer variations were noted.

Results of our investigations, with reservations due to the small sample and its selection, indicate that observer variation is partly responsible for the discrepancies of opinions on (1) the significance of epithelial changes of the cervix; (2) the concept of the development of invasive carcinoma through phases of severe dysplasia to carcinoma in situ; and, consequently, on (3) the efficacy of mass screening in reducing morbidity of and mortality from carcinoma of the cervix.

We wish to thank Professor J. Knowelden, Department of Community Medicine, Sheffield University, for helpful advice; Dr J. Cocker, Derbyshire Royal Infirmary (cyto-histopathologist A) and Dr R. Yule, Christie Hospital and Holt Radium Institute, Manchester (cytologist B) for their cooperation. The survey has been supported by grants from the
Regional Hospital Board, Sheffield, and the British Empire Cancer Campaign.

\section{References}

Ashluwalia, H. S., and Doll, R. (1968). Mortality from cancer of the cervix uteri in British Columbia and other parts of Canada. Brit. J. prev. Med., 22, 161-164.

Boyes, D. A. (1969). The British Columbia screening programme. Obstet. Gynec. Survey, 24, 1005-1014.

Boyes, D. A. Worth, A. J., and Fidler, H. K. (1970). The results of treatment of 4389 cases of preclinical cervical squamous carcinoma. J. Obstet. Gynaec. Brit. Comwlth, 77, 769-780.

Cocker, J., Fox, H., and Langley, F. A. (1968). Consistency in the histological diagnosis of epithelial abnormalities of the cervix uteri. J. clin. Path., 21, 67-70.

Dickinson, L., Mussey, M. E., Soule, E. H., and Kurland, L. T. (1972). Evaluation of the effectiveness of cytologic screening for cervical cancer. Mayo Clin. Proc., 47, 534-544.

Evans, D. M. D. and Sanerkin, N. G. (1970). Cytology screening error rate. In Cytology Automation, edited by D. M. D. Evans, pp. 5-13. Livingstone, Edinburgh and London.

Govan, A. D. T., Haines, R. M., Langley, F. A., Taylor, C. W., and Woodcock, A. S. (1969). The histology and cytology of changes in the epithelium of the cervix uteri. J. clin. Path., 22, 383-395.

Koss, L. G. (1961 and 1968). Diagnostic Cytology and its Histopathological Basis. 1st and 2nd editions. Pitman, London.

Koss, L. G. (1972). Detection of carcinoma of the uterine cervix. J. Amer. med. Ass., 222, 699-700.

Lederer, H., and Lambourne, A. (1973). The results of screenings by cervical cytology and of histological examination of gynaecological operation specimens. J. Obstet. Gynaec. Brit. Comwlth, 80, 67-71.

Macgregor, J. E., Fraser, M. E., and Mann, E. M. F. (1971). Improved prognosis of cervical cancer due to comprehensive screening. Lancet, 1, 74-76.

Martin, P. L. (1972). How preventable is invasive cervical cancer? Amer. J. Obstet. Gynaec., 113, 541-548.

Pedersen, E., Hoeg, K., and Kolstad, P. (1971). Mass screening for carcinoma of the uterine cervix in Østfold county, Norway: an experiment. Acta. obstet. gynaec. scand., Suppl., 11, 5-18.

Seybolt, J. F., and Johnson, W. D. (1971). Cervical cytodiagnostic problems: a survey. Amer. J. Obstet. Gynec., 109, 1089-1103.

Spriggs, A. I. (1972). Population screening by the cervical smear. Nature (Lond.), 238, 135-137.

Wilson, M., Chamberlain, J., and Cochrane, A. L. (1971). Screening for cervical cancer. Lancet, 1, 297-298. 